\title{
Reverse-Link Performance of Synchronous Cellular DS-CDMA Networks in Dispersive Rician Multipath Fading Channels
}

\author{
Seung-Hoon Hwang, Member, IEEE and Lajos Hanzo, Fellow, IEEE \\ School of Electronics and Computer Sciences, University of Southampton, Highfield, Southampton, SO17 1BJ, \\ United Kingdom, Tel.: +44-(0)23-8059-3125, Fax.: +44-(0)23-8059-4508, and Email: 1h@ecs.soton.ac.uk
}

\begin{abstract}
In this paper, the reverse-link performance of synchronous DS-CDMA cellular networks is investigated in Rician multipath fading environments. The system's performance is evaluated in terms of the achievable average bit error rate (BER) and the user capacities of two different network layouts, namely those of a uniform grid of hexagonal multiple cells and a single isolated cell. In the multiple-cell scenario, the impact of the other cells' interference on the attainable capacity of the synchronous DS-CDMA uplink is investigated. Upon comparing both networks to a conventional asynchronous CDMA system, we demonstrate an achievable user capacity gain of $25 \%$ to $\mathbf{5 6} \%$ for synchronous uplink transmissions over that of the corresponding asynchronous transmission scenario at $\mathrm{BER}=10^{-3}$.
\end{abstract}

\section{INTRODUCTION}

The capacity of direct-sequence code-division multiple-access (DS-CDMA) systems is mainly limited by multiple-access interference (MAI), and thus techniques of reducing the MAI, such as multi-user detection, are currently of great interest [1], [2], [3]. In particular, techniques devised for the reverse link have attracted much attention, as the capacity of the reverse link of cellular voice networks is typically lower than that of the forward link [4]. One reason for this is that the spreading code's orthogonality is not maintained, because in the reverse link the arrival times of the signals transmitted by the mobile stations (MSs) are different, given the random geographical distribution of MSs within the cell.

Synchronous DS-CDMA uplink transmission has been proposed for reducing the effects of MAI in terrestrial mobile systems [5], [6], with the additional benefit of having a lower multi-user detection, or interference cancellation complexity than that of asynchronous systems [7]. Therefore, uplink synchronous DS-CDMA is considered an attractive technique for employment in future mobile communication systems [8] or in mobile broadband wireless access [9]. In such applications, synchronous uplink DS-CDMA requires accurate adaptive timing advance control, which is readily achievable in low-mobility indoor and/or pedestrian environments. However, the previous studies in the literature [5], [6] have assumed the presence of Rayleigh fading and neglected the potential performance benefits of having a specular component. In reality, the indoor channel often exhibits a strong line-of-sight (LOS) component [10]. Furthermore, the effects of the asynchronous MAI imposed by the surrounding cells in a

The financial support of the Chevening Trust is gratefully acknowledged. multi-cell environment have not been quantified.

In this paper, the reverse-link performance of synchronous DS-CDMA cellular networks is analyzed in the specific scenario having a direct LOS path, which results in Rician multipath fading. Extending the derivations of [6], the reverse-link performance of synchronous DS-CDMA is evaluated in terms of the achievable average bit error rate (BER) and the attainable capacities of two different network layouts are quantified, namely that of a uniform grid of hexagonal surrounding cells and a single isolated cell. In the multiple-cell scenario, the impact of other-cell interference is investigated for the sake of quantifying the attainable uplink capacity of synchronous DS-CDMA. Upon comparing both networks to conventional asynchronous CDMA, we will demonstrate that synchronous uplink transmissions facilitate higher capacities than asynchronous transmissions.

The paper is organized as follows. In Section II, both the channel and system model are outlined. Our performance analysis of the multiple-cell and single-cell scenario is provided in Section III, while the numerical results are discussed in Section IV. Finally, our concluding remarks are offered in Section V.

\section{Channel And System Model}

We assume that the narrowband modulated signal of each user is first spread by a short orthogonal sequence and then randomized by a pseudo-noise (PN) sequence. Assuming the presence of $K$ active users $(k=1,2, \ldots, K)$, the $k$-th user's transmitted signal is given by

$$
s^{(k)}(t)=\sqrt{2 P_{k}} a(t) W^{(k)}(t) b^{(k)}(t) \cos \left[\omega_{c} t+\phi^{(k)}\right],
$$

where $P_{k}$ is the average transmitted power, $\omega_{c}$ is the common carrier frequency, and $\phi^{(k)}$ is the phase angle of the kth modulator, which is assumed to be uniformly distributed in $[0,2 \pi)$. Furthermore, $a(t)=\sum_{j=-\infty}^{\infty} a_{j} p_{\tau_{c}}\left(t-j T_{c}\right), a_{j} \in\{-1,1\}$ is a $\mathrm{PN}$ randomization sequence, which is common to all the users within a cell [5], where $p_{T_{c}}(t)$ is the rectangular chip-waveform.

The orthogonal channelisation sequence $W^{(k)}(t)$ differentiating all the uplink users is given by

$W^{(k)}(t)=\sum_{j=-\infty}^{\infty} w_{j}^{(k)} p_{T_{w}}\left(t-j T_{w}\right), w_{j}^{(k)} \in\{-1,1\}$

and the data waveform, $b^{(k)}(t)$ of user $\mathrm{k}$ is expressed as 


$$
b^{(k)}(t)=\sum_{j=-\infty}^{\infty} b_{j}^{(k)} p_{T}(t-j T), \quad b_{j}^{(k)} \in\{-1,1\}
$$

In Equations (2) and (3), $T_{k}$. is the chip duration of the user-specific orthogonal uplink sequences and $p_{T}(t)$ is a rectangular pulse of unit height and duration $T$. The PN chip interval $T_{c}$ is related to the data bit interval $T$ by the processing gain $N=T / T_{c}$. For simplicity's sake, we assume that $T_{w}$ is equal to $T_{c}$.

The low-pass impulse response of the band-pass channel of the $k$ th user may be written as [11]

$$
h_{k}(\tau)=\sum_{l=0}^{L^{(k)}-1} \beta_{l}^{(k)} e^{j \theta_{l}^{(k)}} \delta\left[\tau-\tau_{l}^{(k)}\right] .
$$

Each path is characterized by three variables, namely its magnitude $\beta_{l}^{(k)}$, its phase shift $\theta_{l}^{(k)}$ and its propagation delay, $\tau_{i}^{(k)}$. The tapped delay line model describes the frequency selective channel with the aid of the /th multipath delay of the $k$ th user given by $\tau_{l}^{(k)}=\tau_{0}^{(k)}+I T_{c}$ [12]. The Rayleigh fading channel model is modified by adding a known and constant line-of-sight (LOS) component, namely $\alpha e^{j \phi_{\alpha}}$, to the first tap of the tapped delay line representation of the channel. The first path of this model exhibits Rician fading [13]. Assuming that the other paths exhibit Rayleigh fading, the received signal strength of the $k$-th user on the $l$-th propagation path, $l=1, \cdots, L^{(k)}-1$, has a probability density function (pdf) given by [11]

$$
p\left(\beta_{i}^{(k)}\right)=\left(2 \beta_{l}^{(k)} / \Omega_{l}^{(k)}\right) \cdot \exp \left(-\left(\beta_{i}^{(k)}\right)^{2} / \Omega_{l}^{(k)}\right) .
$$

The parameter $\Omega_{l}^{(k)}$ is the second moment of $\beta_{l}^{(k)}$ (i.e., we have $\Omega_{l}^{(k)}=E\left[\left(\beta_{l}^{(k)}\right)^{2}\right]$ ) with $\sum_{l=0}^{\infty} \Omega_{l}=1$, and we assume that $\Omega_{l}^{(k)}$ is related to the second moment of the main tap $\Omega_{0}^{(k)}$ of the negative-exponentially decaying multipath intensity profile (MIP) by

$$
\Omega_{l}^{(k)}=\Omega_{0}^{(k)} e^{-l \delta}, \delta>0, \text { for } 0<l \leq L^{(k)}-1 .
$$

The parameter $\delta$ reflects the decay rate of the average path magnitude as a function of the path delay. Note that a more realistic model is constituted by the negative-exponential MIP, in which the main path conveys more than half of the total received signal power [11], [14], [15].

\section{PERFORMANCE ANALYSIS}

In the synchronous uplink, the arrival time of the first RAKE receiver branch signal in the single-cell scenario is assumed to be synchronous, while the remaining branch signals are asynchronous, since this can be readily achieved by powerful state-of-the-art synchronization techniques [7]. Therefore, we characterise the scenario, in which the arrival times of the paths are modelled as synchronous for the first RAKE receiver branch, but as asynchronous in the rest of the branches. Using the Gaussian approximation method, the MAI terms of the first branch and the rest of the branches are modeled as Gaussian processes having variances equal to the MAI variances for $n=0$ and for $n \geq 1$, respectively. The employment of the Gaussian assumption in the BER calculations is common, since it was found to be quite accurate even for small values of $K(<10)$, provided that the BER is $10^{-3}$ or higher [12]. A coherent RAKE receiver is used, where the number of taps $L_{r}$ is a variable that is less than or equal to $L^{(k)}$. The tap weights and phases are assumed to be perfect estimates of the channel parameters. Perfect power control is assumed. The RAKE receiver finger associated with the first resolvable path is assumed to track only the specular path, while the Rayleigh faded multipath components simply contribute to the interference [13].

\section{A. Multiple-Cell Scenario}

In a multi-cell environment, the effect of asynchronous interference imposed by the surrounding cells should be considered, because it inflicts some performance degradation. The cellular CDMA channel is assumed to be undergoing frequency selective Rician fading superimposed on the fourth-order propagation path loss. We assume encountering the scenario, where $K$ users are supported in each cell. Hence, there are $K-1$ interfering users plus the reference user in the reference cell, and $K$ interfering users in each neighboring cell. The signal received by the reference base station (BS) is expressed as

$$
\begin{aligned}
& r(t)=\sum_{k=1}^{K} \sqrt{2 P \xi\left(r_{k}\right) g\left(r_{k}\right)} \sum_{l=0}^{L^{(k)}-1} \beta_{l}^{(k)} a\left[t-\tau_{l}^{(k)}\right] W^{(k)}\left[t-\tau_{l}^{(k)}\right] \\
& \cdot b^{(k)}\left[t-\tau_{l}^{(k)}\right] \cos \left[\omega_{c} t+\varphi_{l}^{(k)}\right]+m \cdot \sum_{j=1}^{K} \sqrt{2 P \xi\left(r_{j}\right) g\left(r_{j}{ }^{\prime}\right)} \\
& \sum_{l=0}^{L^{(k)}-1} \beta_{l}^{(j)} a\left[t-\tau_{l}^{(j)}\right] W^{(j)}\left[t-\tau_{l}^{(j)}\right] b^{(j)}\left[t-\tau_{l}^{(j)}\right]
\end{aligned}
$$

$\cdot \cos \left[\omega_{c} t+\varphi_{t}^{(j)}\right]+n(t)$,

where we have $\beta_{0}^{(k)}=\alpha, \varphi_{l}^{(k)}$ is the phase of the $l$-th path of the $k$-th carrier and $n(t)$ is the Additive White Gaussian Noise (AWGN) having a double-sided power spectral density of $\eta_{0} / 2$. The first term in (7) represents the signals received from the users within the reference cell, which are located at distances of $r_{k}$ from the BS. The second term represents the interfering signal imposed by the adjacent interfering cells, where $r_{j}{ }^{\prime}$ represents the distances of the interfering users from the reference BS, while $\mathrm{m}$ is the number of adjacent interfering cells considered and $g\left(r_{k}\right)$ is a function of the propagation path loss, which is given as $g\left(r_{k}\right)=1 / r_{k}^{4}$. Furthermore, $\xi(r)=(r / R)^{4}$ is the function describing the effect of the path loss. For the user of interest $(K=1)$, the output of the RAKE receiver is given by

$$
\begin{aligned}
U & =\sum_{n=\hat{l}}^{L_{L}-1} \int_{T_{c}}^{\left(t n T_{c}\right.} r(t) \beta_{n}^{(1)} a\left(t-n T_{c}\right) W^{(1)}\left(t-n T_{n}\right) \cos \left[\omega_{c} t+\varphi_{n}^{(1)}\right] d t \\
& =\sum_{n=0}^{L_{k}-1}\left\{S^{(n)}+I_{m a i}^{(n)-}+I_{s i}^{(n)}+I_{n i}^{(n)}+I_{o c}^{(n)}\right\}
\end{aligned}
$$

where we have

$$
S^{(n)}=\left\{\begin{array}{l}
\sqrt{\frac{P g\left(r_{1}\right)}{2}\left(\frac{r_{1}}{R}\right)^{4}} b_{0}^{(1)} T \alpha^{2}, n=0 \\
\sqrt{\frac{P g\left(r_{1}\right)}{2}\left(\frac{r_{1}}{R}\right)^{4}} b_{0}^{(1)} T\left\{\beta_{n}^{(1)}\right\}^{2}, n \geq 1
\end{array},\right.
$$




$$
\begin{aligned}
& \int \sum_{k=2}^{K} \sum_{l=0}^{L^{(k)}-1} \sqrt{\frac{P g\left(r_{k}\right)}{2}\left(\frac{r_{k}}{R}\right)^{4}} \alpha \beta_{l}^{(k)}\left\{b_{-1}^{(k)} R W_{k 1}\left[\tau_{n i}^{(k)}\right]\right. \\
& I_{m a i}^{(n)}=\left\{\begin{array}{c}
\left.+b_{0}^{(k)} R \hat{W}_{k 1}\left[\tau_{n l}^{(k)}\right]\right\} \cos \left[\varphi_{n i}^{(k)}\right], \quad n=0 \\
\sum_{k=2}^{K} \sum_{l=0}^{L^{(k)}-1} \sqrt{\frac{P g\left(r_{k}\right)}{2}\left(\frac{r_{k}}{R}\right)^{4}} \beta_{n}^{(1)} \beta_{l}^{(k)}\left\{b_{-1}^{(k)} R W_{k 1}\left[\tau_{n l}^{(k)}\right]\right.
\end{array}\right. \\
& \left.+b_{0}^{(k)} R \hat{W}_{k 1}\left[\tau_{n l}^{(k)}\right]\right\} \cos \left[\varphi_{n l}^{(k)}\right], \quad n \geq 1
\end{aligned}
$$

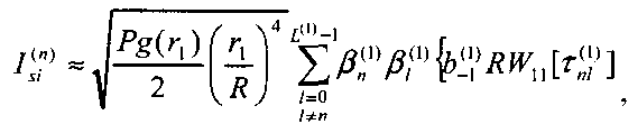

$$
\begin{aligned}
& \left.+b_{-1}^{(1)} R W_{11}\left[\tau_{n i}^{(1)}\right]\right\} \cos \left[\varphi_{n l}^{(1)}\right] \\
& I_{n i}^{(n)}=\int_{n T_{c}}^{\left(t n T_{c}\right.} n(t) \beta_{n}^{(1)} a\left(t-n T_{c}\right) W^{(1)}\left(t-n T_{w}\right) \cos \left[\omega_{c} t+\varphi_{n}^{(1)}\right] d t \\
& I_{o c}^{(n)}=m \cdot \sum_{j=1}^{K} \sum_{l=0}^{L^{(k)}-1} \sqrt{\frac{P g\left(r_{j}{ }^{\prime}\right)}{2}\left(\frac{r_{j}}{R}\right)^{4}} \beta_{n}^{(1)} \beta_{l}^{(j)} \\
& \cdot\left\{b_{-1}^{(j)} R W_{j 1}\left[\tau_{n l}^{(j)}\right]+b_{0}^{(j)} R \hat{W}_{j 1}\left[\tau_{n l}^{(j)}\right]\right\} \cos \left[\varphi_{n l}^{(j)}\right]
\end{aligned}
$$

In the above expressions we have $\beta_{0}^{(1)}=\alpha, b_{0}^{(1)}$ is the information bit to be detected, $b_{-1}^{(1)}$ is the preceding bit, while $\tau_{n l}^{(k)}=\tau_{l}^{(k)}-\tau_{n}^{(1)}$, $\varphi_{n l}^{(k)}=\varphi_{l}^{(k)}-\varphi_{n}^{(1)}$, and $R W$ as well as $\hat{R W}$ are the continuous partial cross-correlation functions of the Walsh-Hadamard PN sequences used in the uplink, which are defined by

$$
R W_{k 1}(\tau)=\int_{0}^{\tau} a(t-\tau) W^{(k)}(t-\tau) a(t) W^{(l)}(t) d t \quad \text { and }
$$$$
R \hat{W}_{k 1}(\tau)=\int^{T} a(t-\tau) W^{(k)}(t-\tau) a(t) W^{(1)}(t) d t, \text { respectively. }
$$

From (8), we see that the output of the $n$-th RAKE receiver branch, $n=0,1, \cdots, L_{r}-1$, consists of five terms. The first term represents the desired signal component to be detected. The second term represents the MAI inflicted by the $(K-1)$ other simultaneous users supported within the reference cell. The third term is the self-interference (SI) imposed by the reference user, while the fourth term represents the AWGN. Finally, the last term in (8e) describes the interference imposed by the other surrounding cells. We assume that the fading statistics of each user are identical, hence the average received power of each resolvable path is the same for all users, yielding $E\left[\left(\beta_{l}^{(k)}\right)^{2}\right]=E\left[\beta_{l}^{2}\right]$.

From (8), the variance of the MAI for $n=0$, conditioned on $\beta_{n}^{(1)}$, can be expressed as follows

$$
\begin{aligned}
\sigma_{\text {mai. } 0}^{2} & =\frac{P}{2} \sum_{k=2}^{K} \sum_{l=0}^{L^{(k)}-1} \alpha^{2} E\left[\left\{\beta_{l}^{(k)}\right\}^{2}\right] E\left[\cos ^{2}\left(\varphi_{0 l}^{(k)}\right)\right] \\
\cdot & E\left[\left\{b_{-1}^{(k)} R W_{k 1}\left(\tau_{0 l}^{(k)}\right)+b_{0}^{(k)} R \hat{W}_{k 1}\left(\tau_{0 l}^{(k)}\right)\right\}^{2}\right] \\
& =\frac{E_{b} T(2 N-3)}{12 N(N-1)} \alpha^{2} \sum_{k=1}^{K} \sum_{i=1}^{L^{(k)}-1} \Omega_{l}^{(k)},
\end{aligned}
$$

where $E_{b}=P T$ is the signal energy per bit. The variance of the MAI for $n \geq 1$ can be formulated as [5]

$$
\sigma_{\text {mai, },}^{2}=\frac{E_{b} T(N-1)}{6 N^{2}}\left\{\beta_{n}^{(1)}\right\}^{2} \sum_{k=2}^{K}\left(\alpha^{2}+\sum_{l=1}^{L^{(k)}-1} \Omega_{l}^{(k)}\right), \quad n \geq 1 .
$$

The conditional variance of $\sigma_{s i, n}^{2}$ is approximated by [8]

$$
\sigma_{s i, n}^{2} \approx \frac{E_{b} T}{4 N}\left\{\beta_{n}^{(1)}\right\}^{l^{\left(L^{(k)}-1\right.}} \sum_{l=1}^{(1)} \Omega_{l},
$$

while the variance of the AWGN is given as

$$
\sigma_{n i, n}^{2}=\frac{T \eta_{0}}{4}\left\{\beta_{n}^{(1)}\right\}^{2}
$$

In a similar fashion to [16], the variance of the other-cell interference term is obtained for a uniform traffic density in the following form

$$
\begin{aligned}
\sigma_{o c, n}^{2} & =\frac{E_{b} T}{6 N}\left\{\beta_{n}^{(1)}\right\}^{2} \sum_{j=1}^{K} \sum_{i=0}^{L^{(k)}-1} \Omega_{l}^{(j)} \iint\left(\frac{r_{j}}{r_{j}{ }^{\prime}\left(r_{j}, \theta\right)}\right)^{4} f\left(r_{j}, \theta\right) d r_{j} d \theta \\
& =\frac{E_{b} T}{6 N}\left(12 \log _{\mathrm{e}}\left(\frac{3}{2}\right)-\frac{19}{4}\right)\left\{\beta_{n}^{(1)}\right\}^{2} \sum_{j=1}^{K}\left(\alpha^{2}+\sum_{i=1}^{L^{(k)}-1} \Omega_{i}^{(j)}\right) .
\end{aligned}
$$

The output of the receiver (conditioned on $\beta_{n}^{(3)}$ ) is a Gaussian random process with a mean given by

$$
U_{s}=\sqrt{\frac{E_{b} T}{2}}\left(\alpha^{2}+\sum_{n=1}^{L_{s}-1}\left\{\beta_{n}^{(1)}\right\}^{2}\right),
$$

while the variance of the total interference is formulated as

$$
\begin{aligned}
& \sigma_{T}^{2}=\sum_{n=0}^{L_{r}-1}\left(\sigma_{\text {mai, }}^{2}+\sigma_{s i, n}^{2}+\sigma_{n i, n}^{2}+\sigma_{o c, n}^{2}\right) \\
& =\left(E_{b} T \Omega_{0}\right)\left\{\frac{\eta_{0}}{4 E_{b} \Omega_{0}}+\frac{K\left(\alpha^{2}+q\left(L^{(k)}, \delta\right)-1\right)}{6 N}\left(12 \log _{e}\left(\frac{3}{2}\right)-\frac{19}{4}\right)\right. \\
& +\frac{q\left(L^{(k)}, \delta\right)-1}{4 N}+\frac{(2 N-3) K\left[q\left(L^{(k)}, \delta\right)-1\right]}{12 N(N-1)} \cdot \frac{\alpha^{2}}{\alpha^{2}+\sum_{n=1}^{L_{r}-1}\left\{\beta_{n}^{(1)}\right\}^{2}}
\end{aligned}
$$$$
\left.+\frac{(N-1)(K-1)\left(\alpha^{2}+q\left(L^{(k)}, \delta\right)-1\right)}{6 N^{2}} \cdot \frac{\sum_{n=1}^{L_{r}-1}\left\{\beta_{n}^{(1)}\right\}^{2}}{\alpha^{2}+\sum_{n=1}^{L_{r}-1}\left\{\beta_{n}^{(3)}\right\}^{2}}\right\}\left(\alpha^{2}+\sum_{n=1}^{L_{r}-1}\left\{\beta_{n}^{(1)}\right\}^{2}\right)
$$

where we have $q\left(L^{(k)}, \delta\right)=\sum_{l=0}^{L^{(k)}-1} e^{-l \delta}=\left(1-e^{-L^{(k)} \delta}\right) /\left(1-e^{-\delta}\right)$.

The probability of error, when encountering a negative-exponential MIP is then yielded as 


$$
\begin{aligned}
P_{e}^{\prime} & =\int_{0}^{\infty} Q\left(\sqrt{\frac{U_{s}^{2}}{\sigma_{T}^{2}}}\right) \cdot p(x) d x \\
& =\int_{0}^{\infty} Q\left(\sqrt{\frac{U_{s}^{2}}{\sigma_{T}^{2}}}\right) \cdot \sum_{k=1}^{L-1} \frac{\pi_{k}}{\Omega_{k}} e^{-x / \Omega_{k}} d x,
\end{aligned}
$$

where $X=\sum_{n=1}^{L_{r}-1}\left\{\beta_{n}^{(3)}\right\}^{2}, Q(x)=(1 / \sqrt{2 \pi}) \int_{x}^{\infty} \exp \left(-u^{2} / 2\right) d u$ and $\pi_{k}=\prod_{\substack{i=1 \\ i \neq k}}^{L-1} \frac{x_{k}}{x_{k}-x_{i}}=\prod_{\substack{i=1 \\ i \neq k}}^{L-1} \frac{\Omega_{k}}{\Omega_{k}-\Omega_{i}}$,

where, the integral in (16) can be readily computed numerically.

\section{B. Single-Cell Scenario}

Assuming $m=0$ in the previous section, we can treat the single cell scenario as a special case of the multiple-cell situation. From (15), the variance of the total interference becomes

$$
\begin{aligned}
& \sigma_{T}^{2}=\frac{E_{b} T(2 N-3)}{12 N(N-1)} \alpha^{2} \sum_{k=2}^{K} \sum_{l=1}^{L^{(k)}-1} \Omega_{l}^{(k)}+\frac{E_{b} T(N-1)}{6 N^{2}} \sum_{n=1}^{L_{r}-1}\left\{\beta_{n}^{(1)}\right\}^{2} \\
& \sum_{k=2}^{K}\left(\alpha^{2}+\sum_{l=1}^{L^{(k)}-1} \Omega_{l}^{(k)}\right)+\frac{E_{b} T}{4 N}\left(\alpha^{2}+\sum_{n=1}^{L_{r}-1}\left\{\beta_{n}^{(l)}\right\}^{2}\right) \sum_{i=1}^{L^{(k)}-1} \Omega_{l}^{(1)} \\
& +\frac{T \eta_{0}}{4}\left(\alpha^{2}+\sum_{n=1}^{L_{r}-1}\left\{\beta_{n}^{(1)}\right\}^{2}\right)
\end{aligned}
$$

and hence the probability of error can be obtained from (16) and (17).

\section{Numerical Results}

In this section, we investigate the effects of the choice of system parameters on the achievable BER performance of a coherent binary phase-shift-keying (BPSK) RAKE receiver in the context of an uplink synchronous DS-CDMA system transmitting over a Rician fading channel exhibiting a negative-exponential MIP. We assume that $L^{(k)}$ is the number of propagation paths that arrive at the receiver of the $k$ th user. The processing gain $N$ and the number $L_{r}$ of RAKE receiver branches are fixed, but we vary the number of simultaneous users $K$. In our performance graphs, $\alpha^{2} / \Omega_{0}$ is the ratio of the specular power to the average power for the first scattered path, a measure which has been used in the previous literature [17]. Indoor radio channel measurements show that Rician distributions tend to have a $K$-factor ranging from $2 \mathrm{~dB}$ to $7 \mathrm{~dB}$ [13]. The effect of different values of $\alpha^{2} / \Omega_{0}$ and $\delta$ on the effective total system capacity will be characterised in Figs. 1 to 2 as well as Tables 1 and 2, where the attainable system performance is evaluated in terms of the achievable average BER and the number of users supported. The BER analysis of an asynchronous CDMA system utilizing maximum ratio combining (MRC) and communicating over both Rayleigh and Rician multipath fading channels can be found in [11], [12] and [17].

Figures 1 and 2 show the achievable average uncoded BER performance as a function of $E_{b} / N_{0}$, when $\alpha^{2} / \Omega_{0}=0 d B$ and $\alpha^{2} / \Omega_{0}=3 d B$ are assumed in conjunction with different MIPs ( $\delta=0.2$ and $\delta=1.0$ ). Measurements carried out by Turin [18] in an urban environment indicate that the MIP is typically negative-exponentially decaying. For characterising the system's performance, we have chosen the parameters of $K=24, N=128$,
$L^{(k)}=L_{r}=3$ and $m=6$. From Figs. 1 and 2, we observe that in the presence of Rician fading, the employment of synchronous uplink DS-CDMA has a more beneficial effect on the achievable system performance, when $\alpha^{2} / \Omega_{0}$ increases to two in conjunction with $\delta=1.0$, than along with $\delta=0.2$. More explicitly, a better BER is achieved, because the reduced multiple access interference encountered by the first path has a higher impact on the achievable system performance, than that of the reduced signal power on the RAKE receiver branches other than the first branch. Intuitively, as the MIP decay factor $\delta$ increases, the received power of the main path including the nonfading LOS component - increases, resulting in a higher specular-signal to noise ratio (SNR). This significantly enhances the achievable performance of the synchronous system. As the specular path power and the MIP decay factor $\delta$ increase, the effect of all the other paths - except for the initial path - becomes negligible as a result of the higher specular SNR and as a consequence of the reduced diversity gain.

The number of users supported by the system, while aiming for a fixed BER, is also an important design criterion in terms of characterising the achievable upper bound of the system's capacity. The number of users supported is summarized to demonstrate the user capacity at $B E R=10^{-3}$ or $10^{-6}$ in Tables 1 and 2 , when we have $E_{b} / N_{0}=10 \mathrm{~dB}$ in conjunction with the MIP decay factors of $\delta=0.2$ and $\delta=1.0$. Comparing Tables 1 and 2 demonstrates that when uplink synchronization is employed, a channel having a negative-exponential MIP in conjunction with $\delta=1.0$ exhibits a higher capacity gain, than that achieved using the MIP decay factor of $\delta=0.2$, while aiming for the same BER value. For the sake of comparison, the achievable performance of the single-cell scenario in conjunction with $m=0$ is also presented. We observe that the characteristics of the cellular environment have a considerable effect on the number of users supported by the system. For example, as seen in Table 1 for $B E R=10^{-3}$, in the single-cell scenario the achievable capacity improvements are around $60 \%$, while in the multiple-cell situation the corresponding improvement decreases to $33 \%$. Nevertheless, the synchronous multi-cell scenario is still capable of achieving a user-capacity gain of about $40 \%$ in comparison to the asynchronous uplink scenario. For the single cell scenario of Table 2, we may observe that the synchronous transmission scenario encountering $\alpha^{2} / \Omega_{0}=0 d B$ shows an even better performance, than the asynchronous system encountering $\alpha^{2} / \Omega_{0}=3 d B$. This implies that maintaining synchronous communications may be expected to be significantly more influential in terms of determining the achievable system performance, when communicating over Rician fading channels, than the value of the fading parameter $\alpha^{2} / \Omega_{0}$.

\section{CONCLUSIONS}

In this paper, we have considered the effects of other-cell interference, that of the direct LOS path's power $\alpha$, as well as the influence of the MIP decay factor on the performance of the synchronous DS-CDMA uplink of cellular networks encountering a Rician multipath-fading channel. The inclusion of a direct LOS path component in the channel model increases the performance differences between the synchronous and asynchronous reverse-link systems. Furthermore, the negative-exponential MIP's decay factor, $\delta$, has a substantial effect on the achievable system performance in a Rician fading channel. Therefore, the BER improvement achieved with the advent of synchronous transmissions depends on the specular path's 
power as well as on the MIP decay factor of the channel, since the effect of all the other paths - except for the main LOS path - becomes negligible as a result of the higher specular SNR and as a consequence of the reduced diversity gain.

In summary, as seen in Tables 1 and 2, for cellular networks communicating over Rician fading channels, the employment of a coherently detected synchronous reverse link shows user capacity improvements ranging from $25 \%$ to $56 \%$ at $\mathrm{BER}=10^{-3}$. Even though a user capacity degradation is encountered in realistic multiple-cell scenarios compared to the single-cell case, the system still exhibits a performance gain for the synchronous DS-CDMA uplink in comparison to the corresponding asynchronous scenario. The performance analysis of a system benefiting from multi-carrier transmissions constitutes the subject of our future research.

\section{REFERENCES}

[1] R.A. Iltis, "Performance of constrained and unconstrained adaptive multiuser detectors for quasi-synchronous CDMA," IEEE Trans. on Commun., Vol. 46, pp. 135-143, Jan. 1998.

[2] H. Sari, F. Vanhaverbeke and M. Moeneclaey, "Extending the capacity of multiple access channets," IEEE Communication Magazine, pp. 74-82, Jan. 2000

[3] H. Sari, F. Vanhaverbeke and M. Moeneclaey, "Multiple access using two sets of orthogonal signal waveforms," IEEE Commun. Letter, Vol. 4, No. 1, pp. 4-6, Jan. 2000

[4] R. Padovani, "Reverse link performance of IS-95 based cellular system," IEEE Personal Commun., vol. 1, no. 3, Third quarter, 1994

[5] E.K. Hong, S.-H. Hwang, K.J. Kim and K.C. Whang, "Synchronous transmission technique for the reverse link in DS-CDMA terrestrial mobile systems," IEEE Trans. on Commun., Vol. 47, pp. 1632-1635, Nov. 1999.

[6] S.-H. Hwang and D.K. Kim, "Performance of reverse-link synchronous DS-CDMA system on a frequency-selective multipath fading channel with imperfect power control," EURASIP Joumal on APP. Sig. Proc, Vol. 8, pp.797-806, Aug. 2002.

[7] L. Hanzo, L.-L. Yang, E.-L. Kuan and K. Yen, Single- and Multicarrier DS-CDMA. John Wiley \& IEEE press, 2003, 1070 pages.

[8] 3GPP TR25.854: Uplink Synchronous Transmission Scheme, 3GPP May 2001 (ftp://ftp.3gpp.org).

[9] IEEE 802.20 C802.20-03/29: Smart antenna and MC-SCDMA, April 2003.

[10] T.S. Rappaport, Wireless communications. Prentice Hall, 1996.

[11] J.G. Proakis, Digital Communications. New York: McGraw-Hill, 1983.

[12] T. Eng and L.B. Milstein, "Coherent DS-CDMA performance in Nakagami multipath fading," IEEE Trans. Commun., Vol. 44, No. 9, pp. 1117-1129, 1996 .

[13] J. Wang and L.B. Milstein, "CDMA overlay situations for microcellular mobile communications," IEEE Trans. on Commun., vol. 43, No. 2/3/4, pp. 603-614, 1995.

[14] D. Parsons, The mobile radio propagation channels. Addison-Wesley, 1992.

[15] Rec. ITU-R TG8-1, "Guideline for evaluation of radio transmission technologies for IMT-2000," Rec. M.1225, 1997.

[16] O.K. Tonguz and M.M. Wang, "Cellular CDMA networks impaired by Rayleigh fading: System performance with power control," IEEE Trans. Veh. Technol., Vol. 43, No. 3, pp. 515-527, 1994.

[17] J.R. Foerster and L.B. Milstein, "Analysis of hybrid, coherent FDMA/CDMA systems in Ricean multipath fading," IEEE Trans. on Commun., vol. 45, pp. 15-18, 1997.

[18] G. Turin, F. Clapp, T. Johnston, S. Fine and D. Lavry, "A statistical model of urban multipath propagation," IEEE Trans. Veh. Technol., Vol. 21, No. I, pp. 1-9, 1972

[19] G.P. Efthymoglou, V.A. Aalo and H. Helmken, "Performance analysis of coherent DS-CDMA systems in a Nakagami fading channel with arbitrary parameters," IEEE Trans. Veh. Technol., Vol. 46, No. 2, pp. 289-297, 1997.

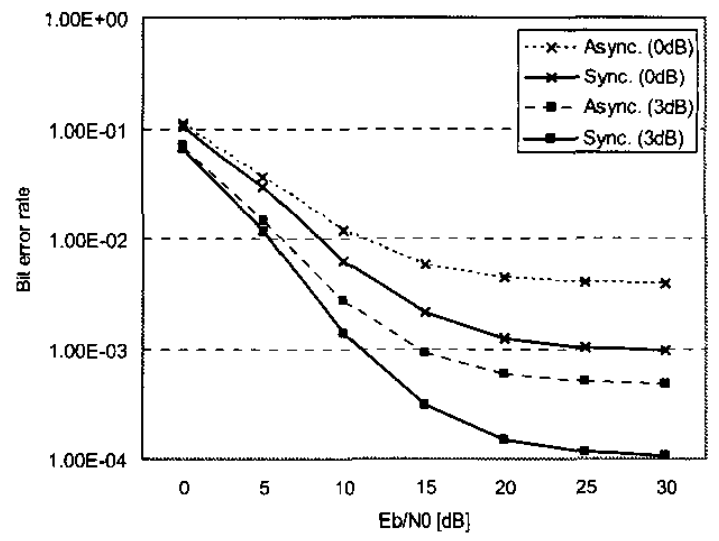

Fig. 1 Uncoded BER vs. Eb/NO in multiple cells $(K=24$, $\alpha^{2} / \Omega_{0}=0$ or $3 d B, \delta=0.2$ )

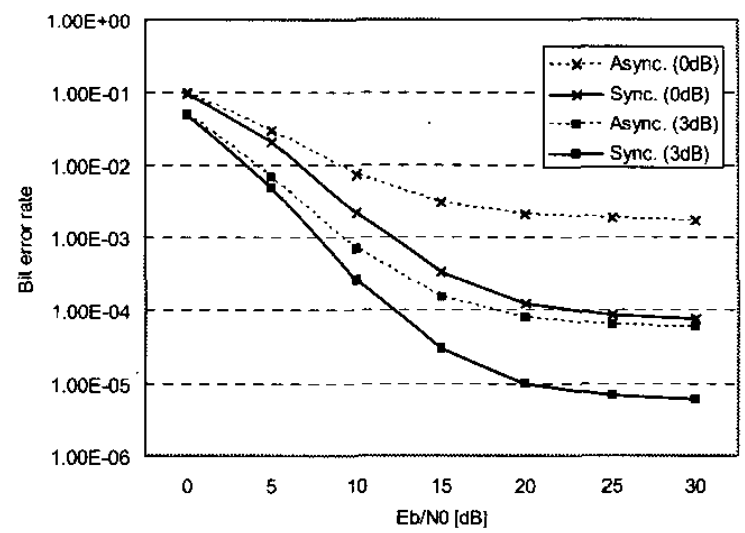

Fig. 2 Uncoded BER vs. Eb/N0 in multiple cells $(K=24$, $\alpha^{2} / \Omega_{0}=0$ or $3 d B, \delta=1.0$ )

\begin{tabular}{|c|c|c|c|c|c|}
\hline \multirow{3}{*}{ BER } & & \multicolumn{4}{|c|}{ USER CAPACITY } \\
\hline & & \multicolumn{2}{|c|}{$\begin{array}{l}\text { MULTIPLE-CELL } \\
\text { SCENARIO }\end{array}$} & \multicolumn{2}{|c|}{$\begin{array}{l}\text { SINGLE-CELLL } \\
\text { SCENARIO }\end{array}$} \\
\hline & $\alpha^{2} / \Omega_{0}$ & $0 \mathrm{~dB}$ & $3 \mathrm{~dB}$ & $0 \mathrm{~dB}$ & $3 \mathrm{~dB}$ \\
\hline \multirow{2}{*}{$\begin{array}{c}10^{-3} \\
\text { (Voice) }\end{array}$} & Sync & 24 & 35 & 42 & 67 \\
\hline & Async & 18 & 28 & 26 & 47 \\
\hline \multirow{2}{*}{$\begin{array}{c}10^{-6} \\
\text { (Data) }\end{array}$} & Sync & $<12$ & 14 & 12 & 25 \\
\hline & Async & $<12$ & $<12$ & $<12$ & 17 \\
\hline
\end{tabular}

TABLE 2 USER CAPACITY. $\left(E_{b} / \eta_{0}=10 d B, \delta=1.0\right.$ )

\begin{tabular}{|c|c|c|c|c|c|}
\hline \multirow{3}{*}{ BER } & & \multicolumn{4}{|c|}{ USER CAPACITY } \\
\cline { 2 - 6 } & & \multicolumn{2}{|c|}{$\begin{array}{c}\text { MULT } \\
\text { SCENE-CELL }\end{array}$} & \multicolumn{2}{c|}{$\begin{array}{c}\text { SINGLE-CELL } \\
\text { SCENARIO }\end{array}$} \\
\cline { 2 - 6 } & $\alpha^{2} / \Omega_{0}$ & $0 \mathrm{~dB}$ & $3 \mathrm{~dB}$ & $0 \mathrm{~dB}$ & $3 \mathrm{~dB}$ \\
\hline $\begin{array}{c}10^{-3} \\
\text { (Voice) }\end{array}$ & Sync & 36 & 48 & 78 & $>84$ \\
\cline { 2 - 6 } & Async & 23 & 37 & 35 & 61 \\
\hline $10^{-6}$ & Sync & 12 & 21 & 30 & 53 \\
\cline { 2 - 6 } (Data) & Async & $<12$ & 15 & 12 & 24 \\
\hline
\end{tabular}

